Pacific Journal of Mathematics

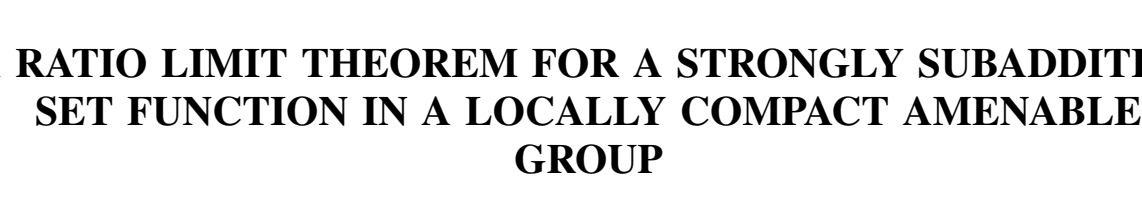




\title{
A RATIO LIMIT THEOREM FOR A STRONGLY SUBADDITIVE SET FUNCTION IN A LOCALLY COMPACT AMENABLE GROUP
}

\author{
J. C. KIEFFER
}

It is the purpose of this paper to prove that the following property holds: Given a locally compact, amenable, unimodular group $G$, if $S$ is a strongly subadditive, nonpositive, right invariant set function defined on the class $\mathscr{K}$ of relatively compact Borel subsets of $G$, and if $\left\{A_{\alpha}\right\}$ is a net in $\mathscr{K}$ satisfying an appropriate growth condition, then

$$
\lim _{\alpha} \lambda\left(A_{\alpha}\right)^{-1} S\left(A_{\alpha}\right)
$$

exists independently of $\left\{A_{\alpha}\right\}$, where $\lambda$ is Haar measure on $G$.

Let $G$ be a locally compact group. Let $\lambda$ be right Haar outer measure defined on the subsets of $G$. Let $\mathscr{K}$ be the class of relatively compact Borel subsets of $G$. If $A$ is a subset of $G$ and $K \in \mathscr{K}$, let $[A]_{K}=\{g \in A: K g \subset A\}=\bigcap_{k \in K \cup\{1\}} k^{-1} A$, where 1 is the identity of $G$. In this paper, we call a locally compact, amenable, unimodular group a lcau group.

Definition 1. Following [1], we define a net $\left\{A_{\alpha}\right\}$ in $\mathscr{K}$ to be a regular net in the locally compact group $G$ if

(D. 1.1) $\lambda\left(A_{\alpha}\right)>0$ for each $\alpha$;

(D. 1.2) $\lim _{\alpha} \lambda\left(K A_{\alpha}\right)^{-1} \lambda\left(\left[A_{\alpha}\right]_{K}\right)=1, K \in \mathscr{K}, K \neq \phi$.

(Even though $K A_{\alpha}$ and $\left[A_{\alpha}\right]_{K}$ may not be Borel measurable, (D. 1.2) makes sense because we required $\lambda$ to be right Haar outer measure, which is defined for all subsets of $G$.)

Lemma 1. A locally compact group $G$ possesses a regular net if and only if $G$ is a lcau group.

Proof. A locally compact group $G$ is amenable if and only if for any $\varepsilon>0$, and for any nonempty compact subset $K$ of $G$, there exists a compact subset $U$ of $G$, of positive measure, such that $\lambda^{*}(U)^{-1} \lambda^{*}(K U)<1+\varepsilon$, where $\lambda^{*}$ is left Haar measure. (See [2].) We call this necessary and sufficient condition for amenability of $G$ condition (A).

Now suppose $G$ possesses a regular net $\left\{A_{\alpha}\right\}$. Then (D.1.2) implies that 


$$
\lim _{\alpha} \lambda\left(K A_{\alpha}\right)^{-1} \lambda\left(A_{\alpha}\right)=1, K \in \mathscr{K}, K \neq \phi .
$$

Taking $K=\{g\}$, where $g$ is any element of $G$, we see that $\Delta(g)=1$. Thus $G$ is unimodular. It then follows that (1) implies condition (A), and thus $G$ is also amenable.

Conversely, suppose now $G$ is lcau. Given $\varepsilon>0$ and a nonempty compact subset $K$ of $G$, we may find by condition (A) a compact set $U=U_{(K, \varepsilon)}$, of positive measure, such that $\lambda(U)^{-1} \lambda\left(K^{2} U\right)<1+\varepsilon$. We direct the set $W=\{(K, \varepsilon): K$ a nonempty compact set in $G, \varepsilon>0\}$ as follows: $\left(K_{1}, \varepsilon_{1}\right)>\left(K_{2}, \varepsilon_{2}\right)$ if and only if $K_{1} \supset K_{2}$ and $\varepsilon_{1}<\varepsilon_{2}$. Then $\left\{V_{(K, \varepsilon)}:(K, \varepsilon) \in W\right\}$ is a regular net of compact subsets of $G$, where $V_{(K, \varepsilon)}=K U_{(K, \varepsilon)}$.

Definition 2. Let $G$ be a regular group. Throughout this paper, we consider a set function $S: \mathscr{C} \rightarrow R$, the set of real numbers, which satisfies the following properties:

(D. 2.1) $S(\phi)=0$.

(D. 2.2) $S$ is strongly subadditive; that is, $S(A \cap B)+S(A \cup B) \leqq$ $S(A)+S(B), A, B \in \mathscr{K}$.

(D. 2.3) $S(A) \leqq 0, A \in \mathscr{K}$.

(D. 2.4) $S(A g)=S(A), A \in \mathscr{K}, g \in G$.

The main result we will prove in this note is the following theorem.

THEOREM 1. Let $G$ be a leau group. Let $S: \mathscr{K} \rightarrow R$ satisfy Definition 2. Then there is an extended real number $r^{*}$ such that $\lim _{\alpha} \lambda\left(A_{\alpha}\right)^{-1} S\left(A_{\alpha}\right)=r^{*}$ for every regular net $\left\{A_{\alpha}\right\}$ in $\mathscr{K}$.

A special case of this theorem, for vector groups, was proved in [7] in order to define entropy in statistical mechanics for classical continuous systems. The theorem can be used to define the entropy of a measurable partition relative to a discrete amenable group of measure-preserving transformations on a probability space, thereby enabling one to generalize the concept of the Kolmogorov-Sinai invariant [5].

One may construct a set function $S$ satisfying Definition 2 as follows: Let $(\Omega, \mathscr{M})$ be a measurable space. For each element $g$ of the regular group $G$, let $T^{g}$ be a measurable transformation from $\Omega$ to $\Omega$. We suppose that $T^{g_{1}} \cdot T^{g_{2}}=T^{g_{1} g_{2}}, g_{1}, g_{2} \in G$. Let $\mathscr{F}$ be a fixed sub-sigmafield of $\mathscr{A}$. If $E$ is a nonempty subset of $G$, let $\mathscr{F}_{E}$ be the smallest sub-sigmafield of $\mathscr{C}$ containing $\bigcup_{g \in E}\left(T^{g}\right)^{-1} \mathscr{F}$. Define $\mathscr{F}_{\phi}=\{\phi, \Omega\}$. Let $P, Q$ be probability measures on $\mathscr{M}$, such that $P$ is stationary with respect to $\left\{T^{g}: g \in G\right\}$ and the fields $\left\{\left(T^{g}\right)^{-1} \mathscr{F}: g \in G\right\}$ 
are independent with respect to $Q$. For each $E \in \mathscr{K}$, let $S(E)$ be the negative of the entropy of $P$ with respect to $Q$ over $\mathscr{F}_{E}$, which we assume finite. The function $S: \mathscr{K} \rightarrow R$ defined in this way can be shown to satisfy Definition 2 in a manner analogous to that employed in [7] for vector groups.

LEMMA 2. If Theorem holds for all sigma-compact lcau groups it holds for all lcau groups.

Proof. Let $d$ be a complete metric on $R^{*}$, the set of extended real numbers, which induces the usual topology on $R^{*}$. Let $\left\{A_{\alpha}\right\}$ be a regular net for a non-sigmacompact lcau group $G$. Suppose $\lim _{\alpha} \lambda\left(A_{\alpha}\right)^{-1} S\left(A_{\alpha}\right)$ does not exist. Then for some $\varepsilon>0$, we may find a sequence $\left\{F_{n}\right\}_{0}^{\infty}$ of elements of $\left\{A_{\alpha}\right\}$ and a sequence $\left\{E_{n}\right\}_{0}^{\infty}$ in $\mathscr{K}$ such that

(a) $F_{0}$ is any $A_{\alpha}$ and $E_{0}$ is an open symmetric neighborhood of the identity.

(b) $d\left(\lambda\left(F_{n}\right)^{-1} S\left(F_{n}\right), \lambda\left(F_{n-1}\right)^{-1} S\left(F_{n-1}\right)\right)>\varepsilon, n \geqq 1$.

(c) $\lambda\left(E_{n-1} F_{n}\right)^{-1} \lambda\left(\left[F_{n}\right]_{E_{n-1}}\right)>1-n^{-1}, n \geqq 1$.

(d) $E_{n}$ is an open symmetric set containing the closure of $\left[E_{n-1} \cup F_{n}\right]^{2}, n \geqq 1$.

Let $G^{\prime}=\bigcup_{n} E_{n}$. It is easily seen that $G^{\prime}$ is an open, sigmacompact subgroup of $G$.

If we restrict $\lambda$ to $G^{\prime}$, we get right Haar measure on $G^{\prime}$. Thus $\left\{F_{n}\right\}$ is a regular sequence for $G^{\prime}$, and $G^{\prime}$ is a lcau group. Assuming Theorem 1 holds for sigma-compact lcau groups, $\lim _{n} \lambda\left(F_{n}\right)^{-1} S\left(F_{n}\right)$ would have to exist, a contradiction of b). Thus $\lim _{\alpha} \lambda\left(A_{\alpha}\right)^{-1} S\left(A_{\alpha}\right)$ exists. Let $\left\{B_{\beta}\right\}$ be another regular net in $G$. Let $s_{1}=\lim _{\alpha} \lambda\left(A_{\alpha}\right)^{-1} S\left(A_{\alpha}\right)$, $s_{2}=\lim \lambda\left(B_{\beta}\right)^{-1} S\left(B_{\beta}\right)$. We show that $s_{1}=s_{2}$. Define sequences $\left\{C_{n}\right\}_{1}^{\infty}$, $\left\{D_{n}\right\}_{1}^{\infty},\left\{E_{n}\right\}_{0}^{\infty}$ in $\mathscr{K}$ such that

(a) $E_{0}$ is an open symmetric neighborhood of the identity, $\left\{C_{n}\right\} \subset$ $\left\{A_{\alpha}\right\},\left\{D_{n}\right\} \subset\left\{B_{\beta}\right\}$.

(b) $\quad d\left(\lambda\left(C_{n}\right)^{-1} S\left(C_{n}\right), s_{1}\right)<n^{-1}, d\left(\lambda\left(D_{n}\right)^{-1} S\left(D_{n}\right), s_{2}\right)<n^{-1}, n \geqq 1$.

(c) $\lambda\left(E_{n-1} C_{n}\right)^{-1} \lambda\left(\left[C_{n}\right]_{E_{n-1}}\right) \geqq 1-n^{-1}, \lambda\left(E_{n-1} D_{n}\right)^{-1} \lambda\left(\left[D_{n}\right]_{E_{n-1}}\right) \geqq 1-$ $n^{-1}, n \geqq 1$.

(d) $E_{n}$ is open, symmetric and contains the closure of $\left[E_{n-1} \cup\right.$ $\left.C_{n} \cup D_{n}\right]^{2}, n \geqq 1$.

It follows that $G^{\prime}=\bigcup_{n} E_{n}$ is an open, sigma-compact, lcau subgroup of $G$ and that $\left\{C_{n}\right\}$ and $\left\{D_{n}\right\}$ are regular sequences for $G^{\prime}$. Therefore, $\lim _{n} \lambda\left(C_{n}\right)^{-1} S\left(C_{n}\right)=\lim _{n} \lambda\left(D_{n}\right)^{-1} S\left(D_{n}\right)$, and so $s_{1}=s_{2}$ by b).

Definition 3. If $G$ is a locally compact group, if $S: \mathscr{K} \rightarrow R$ satisfies Definition 2, and if $A, B \in \mathscr{K}$ with $A \cap B=\phi$, define $S(A \mid B)=$ $S(A \cup B)-S(B)$. 
LEMMA 3. Let $G$ be a locally compact group, and let $S: \mathscr{K} \rightarrow R$ satisfy Definition 2. Then $S$ obeys the following laws:

(L. 3.1) $S(A) \leqq S(B)$ if $A \supset B, A, B \in \mathscr{K}$.

(L. 3.2) If $A_{1}, A_{2}, \cdots, A_{k}$ are elements of $\mathscr{K}$ which partition $A$, then $S(A)=\sum_{i=1}^{k} S\left(A_{i} \mid \bigcup_{j=1}^{i-1} A_{j}\right)$, where an empty union is the null set.

(L. 3.3) $S\left(E \mid D_{1}\right) \leqq S\left(E \mid D_{2}\right), D_{1} \supset D_{2}, E \cap D_{1}=\phi, E, D_{1}, D_{2} \in \mathscr{K}$.

(L. 3.4) $S(E \mid D) \leqq S(E) \leqq 0, E, D \in \mathscr{K}, E \cap D=\phi$.

Proof. (L. 3.2) follows easily from Definition 2. The strong subadditivity of $S$ is equivalent to saying $S(A \backslash B \mid B) \leqq S(A \backslash B \mid A \cap B)$, $A, B \in \mathscr{K}$. Letting $A=E \cup D_{2}$ and $B=D_{1}$, where $E, D_{1}, D_{2}$ satisfy $D_{1} \cap E=\phi$ and $D_{1} \supset D_{2}$, we have $A \cap B=D_{2}$ and $A \backslash B=E$, whence (L. 3.3) follows. In (L. 3.3) if we take $D_{2}=\phi$, (L. 3.4) follows because $S(E \mid \phi)=S(E)$. If $A \supset B$, where $A, B \in \mathscr{K}$, then $S(A)=S(B)+$ $S(A \backslash B \mid B) \leqq S(B)$, and thus (L. 3.1) follows.

Definition 4. We define a locally compact group $G$ to be a $P$ group if there exists for some positive integer $n$ a triple $\left(K,\left\{G_{i}\right\}_{1}^{n},\left\{H_{i}\right\}_{1}^{n}\right)$ such that:

(D. 4.1) $K$ is a nonempty relatively compact Borel set in $G$.

(D. 4.2) $\left\{G_{i}\right\}_{1}^{n}$ and $\left\{H_{i}\right\}_{1}^{n}$ are sequences of closed subgroups of $G$ satisfying $G_{1} \subset H_{1} \subset G_{2} \subset H_{2} \subset \cdots \subset G_{n} \subset H_{n}$.

(D. 4.3) The index of $G_{i}$ in $H_{i}$ is countable, $i=1,2, \cdots, n$.

(D. 4.4) If $E_{i}$ is any set of coset representatives of the right cosets $\left\{G_{i} h: h \in H_{i}\right\}$ of $G_{i}$ in $H_{i}, i=1,2, \cdots, n$, then each $g \in G$ has a unique factorization in the form $g=k e_{1} e_{2} \cdots e_{n}, k \in K, e_{i} \in E_{i}, i=$ $1,2, \cdots, n$. Also, $K\left(\prod_{j=1}^{i-1} E_{j}\right) G_{i}=K\left(\prod_{j=1}^{i-1} E_{j}\right), i=1,2, \cdots, n$, where an empty product is the identity in $G$.

In order to prove Theorem 1 for sigma-compact leau groups, we need to show that such groups are $P$-groups. This we now do, by means of several lemmas. To see how the following lemma may be proved, see [2], page 379 .

LEMMA 4. Let $G^{\prime}$ be a closed normal subgroup of a connected Lie group $G$. Let $\phi: G \rightarrow G / G^{\prime}$ be the canonical homomorphism. Then there exists a map $\tau: G / G^{\prime} \rightarrow G$ such that

(L. 4.1) $\tau$ is a cross-section; that is, $\phi \cdot \tau$ is the identity map on $G / G^{\prime}$.

(L. 4.2) If $U$ is a relatively compact subset of $G / G^{\prime}$, then $\tau(U)$ is a relatively compact subset of $G$.

(L. 4.3) If $U$ is a Borel set in $G / G^{\prime}$ and $V$ is a Borel set in $G^{\prime}$, then $\tau(U) V$ is a Borel set in $G$. 
LEMMA 5. Let $G$ be a connected Lie group and $G^{\prime}$ a closed normal subgroup of $G$ such that $G / G^{\prime}$ is either a vector group or compact. Then if $G^{\prime}$ is a P-group, so is $G$.

Proof. Let $\tau: G / G^{\prime} \rightarrow G$ be the cross-section map provided by Lemma 4. Since $G / G^{\prime}$ is a vector group or compact, it is easy to see that there exists a closed countable subgroup $G^{\prime \prime}$ of $G / G^{\prime}$ and a relatively compact Borel set $K^{\prime}$ in $G / G^{\prime}$ such that $\left\{K^{\prime} g: g \in G^{\prime \prime}\right\}$ partitions $G / G^{\prime}$. If $G^{\prime}$ is a $P$-group with respect to the triple $\left(K,\left\{G_{i}\right\}_{1}^{n}\right.$, $\left.\left\{H_{i}\right\}_{1}^{n}\right)$, then $G$ is a $P$-group with respect to the triple $\left(\tau\left(K^{\prime}\right) K,\left\{G_{i}\right\}_{1}^{n+1}\right.$, $\left.\left\{H_{i}\right\}_{1}^{n+1}\right)$, where $G_{n+1}=G^{\prime}$ and $H_{n+1}=\phi^{-1}\left(G^{\prime \prime}\right)$.

LEMMA 6. If $G$ is a sigma-compact locally compact group and $G^{\prime}$ is an open subgroup of $G$ which is a P-group, then $G$ is a P-group.

Proof. Let $G^{\prime}$ be a $P$-group with respect to the triple ( $K$, $\left.\left\{G_{i}\right\}_{1}^{n},\left\{H_{i}\right\}_{1}^{n}\right)$. Then $G$ is a $P$-group with respect to the triple $\left(K,\left\{G_{i}\right\}_{1}^{n+1}\right.$, $\left.\left\{H_{i}\right\}_{1}^{n+1}\right)$, where $G_{n+1}=G^{\prime}, H_{n+1}=G$.

LEMMA 7. If $G$ is a locally compact group and $G^{\prime}$ is a compact normal subgroup of $G$ such that $G / G^{\prime}$ is a P-group, then $G$ is a $P$ group.

Proof. Suppose $G / G^{\prime}$ is a $P$-group with respect to the triple $\left(K,\left\{G_{i}\right\}_{1}^{n},\left\{H_{i}\right\}_{1}^{n}\right)$. Let $\phi: G \rightarrow G / G^{\prime}$ be the canonical homomorphism. Then $G$ is a $P$-group with respect to the triple $\left(\phi^{-1}(K),\left\{\phi^{-1}\left(G_{i}\right)\right\}_{1}^{n}\right.$, $\left.\left\{\phi^{-}\left(H_{i}\right)\right\}_{1}^{n}\right)$.

THEOREM 2. Every sigma-compact locally compact amenable group is a P-group.

Proof. Every connected amenable Lie group $G$ possesses a series of closed subgroups $G_{0} \subset G_{1} \subset G_{2} \subset \cdots \subset G_{n}=G$, where $G_{0}$ is the identity, $G_{i}$ is normal in $G_{i+1}$, and $G_{i+1} / G_{i}$ is either a vector group or compact, $i=0,1, \cdots, n-1$. (See [3], Theorem 3.3.2, and [4], Lemma 3.3.) Now $G_{0}$ is clearly a $P$-group, so by using Lemma 5 repeatedly we conclude every connected amenable Lie group is a $P$ group. Applying Lemma 6, every sigma-compact amenable Lie group is a $P$-group. For every locally compact group $G$ there exists an open subgroup $G^{\prime}$ of $G$ and a compact normal subgroup $K$ of $G^{\prime}$ such that $G^{\prime} / K$ is a Lie group. (See [6], page 153.) Assuming $G$ in addition is sigma-compact and amenable, so is $G^{\prime} / K$. Thus $G^{\prime} / K$ is a $P$-group and then so is $G^{\prime}$ by Lemma 7 . Then $G$ is a $P$-group by Lemma 6 . 
We fix $G$ to be a sigma-compact lcau group for the rest of the paper. We need to show Theorem 1 holds for $G$. This we accomplish by means of some lemmas and Theorem 3 .

Let $\left(K,\left\{G_{i}\right\}_{1}^{n},\left\{H_{i}\right\}_{1}^{n}\right)$ be a triple with respect to which $G$ is a $P$ group. Let $E_{i}$ be a set of coset representatives of the right cosets of $G_{i}$ in $H_{i}$ such that $1 \in E_{i}, i=1,2, \cdots, n$, where 1 is the identity of $G$. For each $i$, let $\bar{H}_{i}$ be the collection of right cosets of $G_{i}$ in $H_{i}$. (Since $G_{i}$ is not necessarily normal in $H_{i}, \bar{H}_{i}$ need not be a group.) For each $i$, let $\phi_{i}: H_{i} \rightarrow \bar{H}_{i}$ be the map such that $\phi_{i}(h)=G_{i} h, h \in H_{i}$; let $\tau_{i}: \bar{H}_{i} \rightarrow E_{i}$ be the unique map such that $\phi_{i} \cdot \tau_{i}$ is the identity map on $\bar{H}_{i}$. By a total order $<$ on a set $W$, we mean a transitive relation such that for $x, y \in W$ exactly one of the following hold: $x<y, x=y$, or $y<x$. For each $i$, let $<^{i}$ be a total order on $E_{i}$; if $h \in H_{i}$, let $<_{h}^{i}$ be the total order on $E_{i}$ such that if $e, e^{\prime} \in E_{i}$ then $e<_{h}^{i} e^{\prime}$ if and only if $\tau_{i} \cdot \phi_{i}(e h)<{ }^{i} \tau_{i} \cdot \phi_{i}\left(e^{\prime} h\right)$. If $h \in H_{i}$, let $P_{h}^{i}(e)=$ $\left\{e^{\prime} \in E_{i}: e^{\prime}<_{h}^{i} e\right\}$. Let $E=E_{1} E_{2} \cdots E_{n}$. Let $H$ be the locally compact amenable group $H=H_{1} \times H_{2} \times \cdots \times H_{n}$. If $h=\left(h_{1}, h_{2}, \cdots, h_{n}\right) \in H$, let $<_{h}$ be the lexicographical order on $E$ defined as follows: if $e=$ $e_{1} e_{2} \cdots e_{n}$ and $e^{\prime}=e_{1}^{\prime} e_{2}^{\prime} \cdots e_{n}^{\prime}$ are elements of $E$, where $e_{i}, e_{i}^{\prime} \in E_{i}$, then $e<_{h} e^{\prime}$ if and only if there exists an integer $k, n \geqq k \geqq 1$, such that $e_{k}<_{h_{k}}^{h} e_{k}^{\prime}$ and for $n \geqq j>k, e_{j}=e_{j}^{\prime}$. If $h \in H, e \in E$, let $P_{h}(e)=$ $\left\{e^{\prime} \in E: e^{\prime}<_{h} e\right\}$. If $A \in \mathscr{K}, e \in E$, let $\phi_{A}^{e}: H \rightarrow R$ be the function such that $\phi_{A}^{e}(h)=S\left(K e \mid K P_{h}(e) \cap A e\right)=S\left(K \mid K P_{h}(e) e^{-1} \cap A\right), h \in H$.

LEMma 8. If $A \in \mathscr{K}$ and $e \in E$, then $\phi_{A}^{e} \in L^{\infty}(H)$, the space of bounded Borel-measurable real-valued functions with domain $H$.

Proof. Fix $A \in \mathscr{K}, e \in E$. By (L. 3.4), $\phi_{A}^{e} \leqq 0$. To achieve a lower bound, let $E^{\prime}=\left\{e^{\prime} \in E: K e^{\prime} \cap A e \neq \phi\right\}$. Since $K E^{\prime} \subset K K^{-1} A e, E^{\prime}$ is finite. Let $F=\{e\} \cup E^{\prime}$. By (L. 3.2), $S(K F)=\sum_{f \in F} S\left(K f \mid K P_{h}(f) \cap\right.$ $K F$ ). By (L. 3.3) and (L. 3.4), $S(K F) \leqq S\left(K e \mid K P_{h}(e) \cap K F\right) \leqq$ $S\left(K e \mid K F_{h}(e) \cap A e\right)=\phi_{A}^{e}(h)$, where the fact that $K F \supset A e$ was used. Thus $\phi_{A}^{e}$ is a bounded function. We now show that it is a Borel measurable function. It is easily seen that $\phi_{A}^{e}$ is a simple function with possible values $S\left(K e \mid K F^{\prime} \cap A e\right), F^{\prime \prime} \subset F$. If $F^{\prime \prime} \subset F$, then $\phi_{A}^{e}=S\left(K e \mid K F^{\prime} \cap A e\right)$ on the set $\left\{h \in H: P_{h}(e) \cap F=F^{\prime}\right\}$, which is equal to the intersection of the sets $\bigcap_{f \in F^{\prime}}\left\{h: f \in P_{h}(e)\right\}$ and $\bigcap_{f \in F \backslash F^{\prime}}\left\{h: f \notin P_{h}(e)\right\}$. Thus $\phi_{A}^{e}$ is Borel measurable if for each $f \in F,\left\{h \in H: f \in P_{h}(e)\right\}$ is a Borel set. If $f=e$, this set is empty. Thus, fix $f \in F, f \neq e$. Let $f=f_{1} f_{2} \cdots f_{n}$, and $e=e_{1} e_{2} \cdots e_{n}$, where $e_{i}, f_{i} \in E_{i}$ for each $i$. Let $j=\max \left\{i: f_{i} \neq e_{i}\right\}$. Then $\left\{h \in H: f \in P_{h}(e)\right\}=\left\{h \in H: f_{j} \in P_{h_{j}}^{j}\left(e_{j}\right)\right\}$, where $h_{j} \in H_{j}$ is the $j^{\text {th }}$ component of $h \in H$. This is a Borel set in $H$ if $\left\{h \in H_{j}: f_{j} \in P_{h}^{j}\left(e_{j}\right)\right\}$ is a Borel set in $H_{j}$. Now this latter set is the union of the sets $\left\{h \in H_{j}: G_{j} f_{j} h=G_{j} g_{1}, G_{j} e_{j} h=G_{j} g_{2}\right\}$ where $\left(g_{1}, g_{2}\right)$ ranges over all ordered 
pairs such that $g_{1}, g_{2} \in E_{j}$ and $g_{1}<^{j} g_{2}$. Since the union is a countable union of closed subsets of $H_{j}$, Borel measurability follows.

Lemma 9. Let $\mu$ be a left invariant mean on $L^{\infty}(H)$. Then $\mu\left(\phi_{A}^{e}\right)=\mu\left(\phi_{A}^{1}\right), A \in \mathscr{K}, e \in E$.

Proof. Fix $A \in \mathscr{K}, e \in E$. We observe that

$$
\begin{aligned}
K P_{h}(e) e^{-1} & =\left[\bigcup_{i=1}^{u} K\left(\prod_{j=1}^{i-1} E_{j}\right) P_{h_{i}}^{i}\left(e_{i}\right) e_{i+1} \cdots e_{n}\right] e^{-1} \\
& =\bigcup_{i=1}^{n}\left[K\left(\prod_{j=1}^{i-1} E_{j}\right) G_{i} P_{h_{i}}^{i}\left(e_{i}\right) e_{i}^{-1} \cdots e_{2}^{-1} e_{1}^{-1}\right],
\end{aligned}
$$

by (D. 4.4), where $h=\left(h_{1}, h_{2}, \cdots, h_{n}\right) \in H$ and $e=e_{1} e_{2} \cdots e_{n}$. It is routine to show that $G_{i} P_{h_{i}}^{i}\left(e_{i}\right)=G_{i} P_{1}^{i}\left(\tau_{i} \cdot \phi_{i}\left(e_{i} h_{i}\right)\right) h_{i}^{-1}$. Also, since $e_{j} \in G_{i}$ for $j<i$, we have $\phi_{i}\left(e_{i} h_{i}\right)=\phi_{i}\left(e_{1} e_{2} \cdots e_{i} h_{i}\right)$. Thus, $K P_{h}(e) e^{-1}=$ $\bigcup_{i=1}^{n}\left[K\left(\prod_{j=1}^{i-1} E_{j}\right) P_{1}^{i}\left(\tau_{i} \cdot \phi_{i}\left(e_{1} \cdots e_{i} h_{i}\right)\right)\left(e_{1} \cdots e_{i} h_{i}\right)^{-1}\right]=K P_{m h}(1)$, where $m=$ $\left(m_{1}, m_{2}, \cdots, m_{n}\right) \in H$ satisfies $m_{i}=\prod_{j=1}^{i} e_{j}, i=1,2, \cdots, n$. Thus $\phi_{A}^{e}(h)=$ $\phi_{A}^{1}(m h), h \in H$, from which the lemma follows.

THEOREM 3. Let $\left\{A_{\alpha}\right\}$ be a regular net in the sigmacompact lcau group G. Then $\lim _{\alpha} \lambda\left(A_{\alpha}\right)^{-1} S\left(A_{\alpha}\right)=\inf _{B \in \mathscr{K}} \lambda(K)^{-1} \mu\left(\phi_{B}^{1}\right)$.

Proof. Fix the regular net $\left\{A_{\alpha}\right\}$. Now $K E_{\alpha}^{\prime} \subset A_{\alpha} \subset K E_{\alpha}$, where $E_{\alpha}=\left\{e \in E: K e \cap A_{\alpha} \neq \phi\right\}, E_{\alpha}^{\prime}=\left\{e \in E: K e \subset A_{\alpha}\right\}$. Thus by (L.3.1), $S\left(K E_{\alpha}\right) \leqq S\left(A_{\alpha}\right) \leqq S\left(K E_{\alpha}^{\prime}\right)$. We show that $\lim \sup _{\alpha} \lambda\left(A_{\alpha}\right)^{-1} S\left(K E_{\alpha}^{\prime}\right) \leqq L$ and $\lim \inf _{\alpha} \lambda\left(A_{\alpha}\right)^{-1} S\left(K E_{\alpha}\right) \geqq L$, where $L=\inf _{B \in \mathscr{K}} \lambda(K)^{-1} \mu\left(\phi_{B}^{1}\right)$. Now $S\left(K E_{\alpha}\right)=\sum_{e \in E_{\alpha}} S\left(K e \mid K P_{h}(e) \cap K E_{\alpha}\right) \geqq \sum_{e \in E_{\alpha}} \phi_{B_{\alpha}}^{e}$, where $B_{\alpha}=\bigcup_{e \in E_{\alpha}}$ $K E_{\alpha} e^{-1}$. Applying $\mu$ to the inequality and using Lemma $9, S\left(K E_{\alpha}\right) \geqq$ $\left|E_{\alpha}\right| \mu\left(\phi_{B_{\alpha}}^{1}\right) \geqq\left|E_{\alpha}\right| \lambda(K) L=\lambda\left(K E_{\alpha}\right) L$, where $\left|E_{\alpha}\right|$ denotes the cardinality of $E_{\alpha}$. Since $K E_{\alpha} \subset K K^{-1} A_{\alpha}$ we have $\lim _{\alpha} \lambda\left(A_{\alpha}\right)^{-1} \lambda\left(K E_{\alpha}\right)=1$, by the regularity of $\left\{A_{\alpha}\right\}$. Thus $\liminf _{\alpha} \lambda\left(A_{\alpha}\right)^{-1} S\left(K E_{\alpha}\right) \geqq L$. Fix $B \in \mathscr{K}$. We suppose that $B \supset K$. Now $S\left(K E_{\alpha}^{\prime}\right)=\sum_{e \in E_{\alpha}^{\prime}} S\left(K e \mid K P_{h}(e) \cap K E_{\alpha}^{\prime}\right) \leqq$ $\sum_{e \in F_{\alpha}} \phi_{B}^{e}$ where $F_{\alpha}=\left\{e \in E_{\alpha}^{\prime}: K E_{\alpha}^{\prime} e^{-1} \supset B\right\}$. Applying $\mu, S\left(K E_{\alpha}^{\prime}\right) \leqq$ $\lambda\left(K F_{\alpha}\right) \lambda(K)^{-1} \mu\left(\phi_{B}^{1}\right)$. We could conclude that lim $\sup _{\alpha} \lambda\left(A_{\alpha}\right)^{-1} S\left(K E_{\alpha}^{\prime}\right) \leqq L$, provided $\lim _{\alpha} \lambda\left(A_{\alpha}\right)^{-1} \lambda\left(K F_{\alpha}\right)=1$. This limit is one by the regularity of $\left\{A_{\alpha}\right\}$, since $\left[A_{\alpha}\right]_{K K^{-1} B K^{-1}} \subset K F_{\alpha}$. To see this, let $x \in\left[A_{\alpha}\right]_{K K^{-1} B K^{-1}}$. By definition, $K K^{-1} B K^{-1} x \subset A_{\alpha}$. Now $x \in K e$ for some $e \in E$. We have $K e \subset K K^{-1} x \subset K K^{-1} B K^{-1} x \subset A_{\alpha}$. Thus $e \in E_{\alpha}^{\prime}$. It will follow that $x \in K F_{\alpha}$ if $B e \subset K E_{\alpha}^{\prime}$. To see this, let $y \in B e$. Then $y \in K e^{\prime}$ for some $e^{\prime} \in E$. Now $K e^{\prime} \subset K K^{-1} y \subset K K^{-1} B e \subset K K^{-1} B K^{-1} x \subset A_{\alpha}$. Thus $e^{\prime} \in E_{\alpha}^{\prime}$ and $y \in K E_{\alpha}^{\prime}$. 


\section{REFERENCES}

1. M. F. Driscoll, J. N. McDonald, and N. A. Weiss, LLN for weakly stationary processes on locally compact abelian groups, Ann. of Prob., 2 (1974), 1168-1171.

2. W. R. Emerson and F. P. Greenleaf, Covering properties and Folner conditions for locally compact groups, Math. Zeitschr., 102 (1967), 370-384.

3. F. P. Greenleaf, Invariant Means on Topological Groups, Van Nostrand, 1969.

4. K. Iwasawa, On some types of topological groups, Ann. of Math., 50 (1949), 507558.

5. J. C. Kieffer, An entropy equidistribution property for a measurable partition under the action of an amenable group, Bull. Amer. Math. Soc., 81 (1975), 464-466.

6. D. Montgomery and L. Zippin, Topological transformation groups, Interscience, 1955.

7. D. W. Robinson and D. Ruelle, Mean entropy of states in classical statistical meehanics, Commun. Math. Phys., 5 (1967), 288-300.

Received May 15, 1975.

UNIVERSITY OF MISSOURI-ROLLA 


\section{PACIFIC JOURNAL OF MATHEMATICS}

\section{EDITORS}

RICHARD ARENS (Managing Editor) University of California

Los Angeles, California 90024

\section{R. A. BEAUMONT}

University of Washington Seattle, Washington 98105
J. DugundjI Department of Mathematics University of Southern Californı Los Angeles, California 90007

D. Gilbarg AND J. Milgram Stanford University Stanford, California 94305

\section{ASSOCIATE EDITORS}

E. F. BECKENBACH

B. H. NeUMANN

F. WOLF

K. YosHidA

\section{SUPPORTING INSTITUTIONS}

UNIVERSITY OF BRITISH COLUMBIA CALIFORNIA INSTITUTE OF TECHNOLOGY UNIVERSITY OF CALIFORNIA MONTANA STATE UNIVERSITY UNIVERSITY OF NEVADA NEW MEXICO STATE UNIVERSITY OREGON STATE UNIVERSITY UNIVERSITY OF OREGON OSAKA UNIVERSITY

\author{
UNIVERSITY OF SOUTHERN CALIFORNIA \\ STANFORD UNIVERSITY \\ UNIVERSITY OF TOKYO \\ UNIVERSITY OF UTAH \\ WASHINGTON STATE UNIVERSITY \\ UNIVERSITY OF WASHINGTON \\ AMERICAN MATHEMATICAL SOCIETY \\ NAVAL WEAPONS CENTER
}




\section{Pacific Journal of Mathematics}

\section{Vol. 61, No. $1 \quad$ November, 1975}

Jiří Adámek, V. Koubek and Věra Trnková, Sums of Boolean spaces represent every

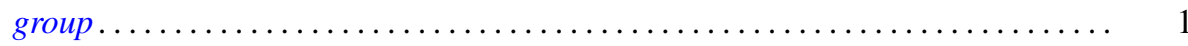

Richard Neal Ball, Full convex l-subgroups and the existence of $a^{*}$-closures of

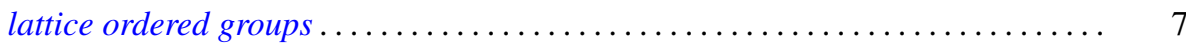

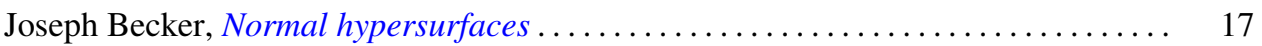

Gerald A. Beer, Starshaped sets and the Hausdorff metric . . . . . . . . . . . . . 21

Dennis Dale Berkey and Alan Cecil Lazer, Linear differential systems with

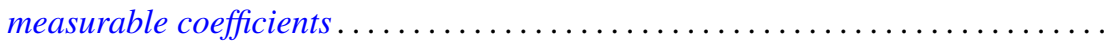

Harald Boehme, Glättungen von Abbildungen 3-dimensionaler

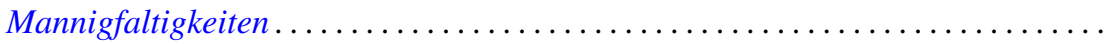

Stephen LaVern Campbell, Linear operators for which $T^{*} T$ and $T+T^{*}$

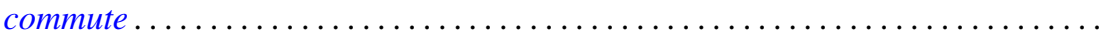

H. P. Dikshit and Arun Kumar, Absolute summability of Fourier series with

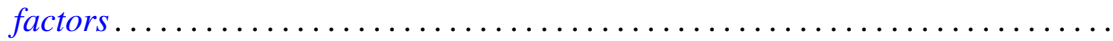

Andrew George Earnest and John Sollion Hsia, Spinor norms of local integral

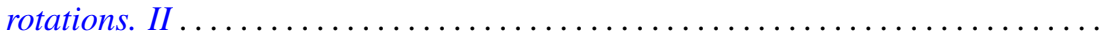

Erik Maurice Ellentuck, Semigroups, Horn sentences and isolic structures .........

Ingrid Fotino, Generalized convolution ring of arithmetic functions . . . . . . . . . . .

Michael Randy Gabel, Lower bounds on the stable range of polynomial rings .......

Fergus John Gaines, Kato-Taussky-Wielandt commutator relations and

characteristic curves

Theodore William Gamelin, The polynomial hulls of certain subsets of $C^{2}$

R. J. Gazik and Darrell Conley Kent, Coarse uniform convergence spaces. . .

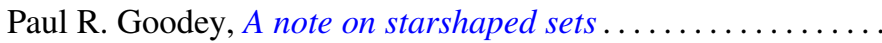

Eloise A. Hamann, On power-invariance

M. Jayachandran and M. Rajagopalan, Scattered compactification for $N \cup\{P\}$. . .

V. Karunakaran, Certain classes of regular univalent functions .

John Cronan Kieffer, A ratio limit theorem for a strongly subadditive set function in a locally compact amenable group .................

Siu Kwong Lo and Harald G. Niederreiter, Banach-Buck measure, density, and uniform distribution in rings of algebraic integers ........

Harold W. Martin, Contractibility of topological spaces onto metric spaces ....

Harold W. Martin, Local connectedness in developable spaces .

A. Meir and John W. Moon, Relations between packing and covering numbers of a tree.

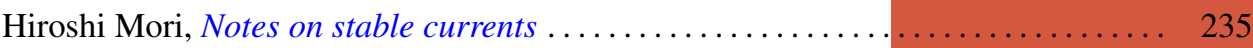

Donald J. Newman and I. J. Schoenberg, Splines and the logarithmic function . . . . 241

M. Ann Piech, Locality of the number of particles operator....

Fred Richman, The constructive theory of $K T$-modules .......

Gerard Sierksma, Carathéodory and Helly-numbers of

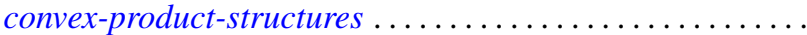

Raymond Earl Smithson, Subcontinuity for multifunctions .... . . 